nation mit Levetiracetam zeigte keinen zusätzlichen Nutzen, der kann aber bei einer wegen mangelnder Wirksamkeit/Verträglichkeit erfolglosen Vorbehandlung mit Levetiracetam durchaus gegeben sein.

Bei geringem Interaktionspotenzial mit dem Cytochrom P 450-System sind klinisch relevante Interaktionen mit anderen Antiepileptika oder oralen Kontrazeptiva nicht bekannt. Brivaracetam steht in drei Formulierungen zur Verfügung: als Filmtablette in den Do- sierungen 10, 25, 50, 75 und $100 \mathrm{mg}$, als Lösung zum Einnehmen (z. B. bei Schluckstörungen) und als intravenöse Lösung unter anderem für Notfälle, auf der Intensivstation und für Patienten, die sich einer Operation unterziehen müssen.

\section{Dr. Matthias Herrmann}

Presse-Round-Table „Brivaracetam: eine neue Therapieoption für Epilepsie-Patienten mit unkontrollierten Anfällen“, Berlin, 16.1.2016; Veranstalter: UCB Pharma

\title{
MS - Therapieziele regelmäßig im Blick haben
}

— Die Auswahl an Wirkstoffen für die Therapie der schubförmig remittierenden Multiplen Sklerose (RRMS) hat in den letzten Jahren deutlich zugenommen. Wie die Therapieentscheidung in der klinischen Praxis gefällt wird, erläuterte Dr. Christian Menges, Facharzt für Neurologie und Psychiatrie, anhand von Beispielen aus seiner Schwerpunktpraxis für MS und Parkinson in Ludwigshafen. Neben dem Ziel Freiheit von Krankheitsaktivität („,no evidence of disease activity", NEDA) rücken in der RRMS-Therapie vor allem patientenindividuelle Faktoren in den Vordergrund. „Für den Arzt ist eine frühzeitige Behandlung mit möglichst wirksamen Medikamenten oberstes Ziel, den Patienten ist vor allem die Erhaltung ihrer Lebensqualität wichtig", erläuterte Menges. Um individuelle Patientenwünsche berücksichtigen zu können, bedarf es einer Abwägung zwischen der Belastung und den Risiken der Krankheit und denen der einzuleitenden Therapie.

Eine Langzeitanalyse über sechs Jahre zeigte ein günstiges Nutzen-Risiko-Profil für Dimethylfumarat (DMF, Tecfidera ${ }^{\circledR}$ ) bei RRMS-Patienten [Pozzilli $C$ et al. ECTRIMS 2015, Poster P544]: Die mittlere Schubrate bei neu diagnostizierten Patienten, die durchgängig DMF erhielten, lag bei 0,14. $84,3 \%$ der Patienten unter kontinuierlicher Therapie waren ohne Behinderungsprogression. Auch immunmodulatorisch vorbehandelte Patienten profitierten von der DMF-Therapie. Es wurde kein erhöhtes Risiko für schwere opportunistische Infektionen unter DMF beobachtet, mit Ausnahme der sehr seltenen Fälle einer progressiven multifokalen Leukenzephalopathie (PML) bei anhaltender Lymphopenie. Deshalb ist vor Therapiebeginn mit DMF ein großes Blutbild inklusive Lymphozytenwert Pflicht. Auch Leber- und Nierenfunktion sollten überprüft werden. Unter der Therapie ist laut Fachinformation eine Kontrolle der Lymphozytenzahl alle drei Monate vorgesehen. Bei einer anhaltenden Lymphopenie von $<500 / \mu$ über sechs Monate empfiehlt Professor Ralf Gold, Direktor der Neurologischen Universitätsklinik Bochum, eine Unterbrechung der Behandlung, um das PML-Risiko möglichst gering zu halten.

Mit der Verfügbarkeit moderner Therapieoptionen wie DMF ist eine erhöhte Wachsamkeit beim Therapieverlauf angezeigt um rechtzeitig zu optimieren. Eine regelmäBige Prüfung der Läsionslast im MRT ist ratsam. Schubaktivität und Behinderungsprogression lassen sich ein bis zweimal im Jahr vor allem im Patientengespräch analysieren, so dass frühzeitig erkannt werden kann, ob die aktuelle Therapie noch zielführend ist und zu den Bedürfnissen der Patienten passt oder ob sie optimiert werden sollte. „Man muss die Patienten sensibilisieren, damit sie Veränderungen wahrnehmen“, erklärte Menges. „Neben dem übergeordneten Ziel Freiheit von Krankheitsaktivität liegt die besondere Herausforderung in der Behandlung der RRMS darin, eine Therapie zu finden, mit der sich die Patienten identifizieren, sodass sie diese zuverlässig fortführen. Eine Immunmodulation ist für den Patienten schließlich nicht direkt spürbar", sagte Menges abschließend.

\section{Julia Rustemeier, Springer Medizin}

Pressekonferenz Zwei Jahre Tecfidera ${ }^{\oplus}$ - Veränderung der Therapiestandards der RRMS, München, 23.2.2016; Veranstalter: Biogen

\section{Exklusiver AOK-Partner}

Die Glenmark Arzneimittel GmbH erhält bei der Ausschreibung der AOK den exklusiven Zuschlag für die Wirkstoffe Riluzol (Glentek) und in Bietergemeinschaft für Pramipexol (Glepark) für die Vertragslaufzeit vom 1.4.2016 bis 31.3.2018.

Nach Informationen von Glenmark

\section{Diabetische Neuropathie}

Laut der PROTECT-Studie [Ziegler $\mathrm{D}$ et al. J Diabetes Complications 2015; 29: 998 - 1002] besteht bei über $50 \%$ der Typ-2-Diabetiker ein Neuropathieverdacht. Zwei Drittel von ihnen wussten vorher nichts davon. Mit der Aufklärungsinitiative „Diabetes! Hören Sie auf Ihre Füße?" informiert Wörwag zusammen mit der Deutschen Diabetes Stiftung über die diabetische Neuropathie, um deren Früherkennung bei Diabetikern zu fördern.

Nach Informationen von Wörwag Pharma

\section{Opiatsubstitution}

Zur Behandlung opiat-/opioidabhängiger Patienten im Rahmen der Substitutionstherapie steht die L-Polamidon ${ }^{\circledR}$ Tablette in den Wirkstärken 5 und $20 \mathrm{mg}$ zur Verfügung. Sie ist eine Ergänzung zur L-Polamidon ${ }^{\oplus}$-Lösung, beide Formulierungen enthalten den Wirkstoff Levomethadon. Take-Home-Patienten profitieren vom vereinfachten Handling der Tabletten.

Nach Informationen von Sanofi

\section{Mehr Lebensqualität für Parkinson-Patienten}

Mit Safinamid (Xadago ${ }^{\odot}$ ) steht seit Mai 2015 eine hocheffektive und gut verträgliche Medikation mit einem gänzlich neuen pharmakologischen Wirkprinzip für die Add-on-Therapie von Patienten im mittleren bis späten Stadium der Parkinson-Erkrankung zur Verfügung. In den zulassungselevanten klinischen Studien verbesserte Safinamid als Begleittherapie zu L-Dopa die motorischen Symptome, verlängerte die tägliche Zeit im On und erhöhte die Lebensqualität auch in der Langzeitanwendung.

Nach Informationen von Zambon 